American J. of Engineering and Applied Sciences 3 (1): 201-206, 2010

ISSN 1941-7020

(C) 2010 Science Publications

\title{
Hardware Implementation of Truncated Multipliers Using Spartan-3AN, Virtex-4 and Virtex-5 FPGA Devices
}

\author{
Muhammad H. Rais \\ Department of Electrical Engineering, College of Engineering, \\ King Saud University, Kingdom of Saudi Arabia
}

\begin{abstract}
Problem statement: The development cost for Application Specific Integrated Circuits (ASICs) are high, algorithms should be verified and optimized before implementation. The Digital Signal Processing (DSP), image processing and multimedia requires extensive use of multiplication. The truncated multipliers can easily be implemented using Field Programmable Gate Array (FPGA) devices. Approach: This research presented the comparative study of Spartan-3AN, Virtex-4 and Virtex-5 FPGA devices. The implementation of standard and truncated multipliers is done using Very high speed integrated circuit Hardware Description Language (VHDL). Results: Remarkable reduction in FPGA resources, delay and power was achieved using truncated multipliers instead of standard parallel multipliers when the full precision of the standard multiplier is not required. The three devices showed significant improvement for truncated multipliers as compared to standard multipliers. Results showed that the anomaly in Spartan-3AN average connection and maximum pin delay have been efficiently reduced in Virtex-4 and Virtex-5 devices. Conclusion: The Virtex-5 FPGA device showed better performance as compared to Spartan-3AN and Virtex-4 FPGA devices.
\end{abstract}

Key words: Digital Signal Processing (DSP), Field Programmable Gate Array (FPGA), Spartan-3AN, truncated multiplier, VHDL, Virtex-4, Virtex-5

\section{INTRODUCTION}

Multiplication is a core operation in many algorithms used in scientific computations such as Digital Signal Processing (DSP). Over the years the computational complexities of algorithms used in Digital Signal Processors (DSPs) have been gradually increased. Therefore, DSP requires fast and efficient parallel multipliers for general purpose as well as application specific architectures.

In many cases implementation of DSP algorithm demands using Application Specific Integrated Circuits (ASICs). In particular, if the processing has to be performed under real time conditions, such algorithms have to deal with high throughput rates. This is especially required for image processing applications. Since development costs for ASICs are high, algorithms should be verified and optimized before implementation.

Nevertheless, Very Large Scale Integration (VLSI) technology has grown up to such stage that a hardware implementation has become a desirable alternative. Significant speedup in computation time can be achieved by assigning computation intensive tasks to hardware and by exploiting the parallelism in algorithms. Recently, Field Programmable Gate Arrays
(FPGAs) have emerged as a platform of choice for efficient hardware implementation of computation intensive algorithms (Maxfield, 2004). FPGA have the benefit of hardware speed and the flexibility of software. FPGAs enable a high degree of parallelism and can achieve orders of magnitude speedup over General Purpose Processors (GPPs). This is a result of increasing embedded resources available on FPGA. The three main factors that play an important role in FPGA based design are the targeted FPGA architecture, Electronic Design Automation (EDA) tools and design techniques employed at the algorithmic level using hardware description languages. In FPGAs, the choice of the optimum multiplier involves three key factors: area, propagation delay and reconfiguration time. Therefore, FPGA has become viable technology and an attractive alternative to ASICs (Maxfield, 2004; Todman et al., 2005).

Applications such as DSP, image processing and multimedia require extensive use of multiplication and squaring functions (Sheu and Lin, 2002; Walters et al., 2003). A full width digital $n \times n$ multiplier computes the $2 \mathrm{n}$ output as a weighted sum of partial products (Baugh and Wooley, 1973). If the product is truncated to n-bits, the least-significant columns of the product matrix contribute little to the final result. To take advantage of 
this, truncated multipliers and squarers do not form all of the least-significant columns in the partial-product matrix (Stine and Duverne, 2003; Swartzlander, 1999). By eliminating more columns the area and power consumption of the arithmetic unit are significantly reduced and in many cases the delay also decreases. Though, truncating the multiplier matrix introduces additional error into the computation.

Cryptography applications, also requires not only a significant number of multiplication and squaring functions but also large integers (Stallings, 2006). Achieving efficient realization of the multiplication may have a significant impact on the specific applications in terms of speed, power dissipation and area. Many research efforts have been presented in literature to achieve hardware efficient implementation of a truncated multiplier. The basic idea of these techniques is to discard some of the less significant partial products and to introduce a compensation circuit that partly compensates for the dropped terms, thereby reducing approximation error (Jou et al., 1999; Van et al., 2000; Kidambi et al., 1996; Strollo et al., 2005; Lim, 1992; Kuang and Wang, 2006). Garofalo et al. (2008) presented a truncated multiplier with minimum square error for every inputs' bit width. Rais (2009a; 2009b) presented design and implementation of fixed width standard and truncated multipliers using FPGA devices.

Truncated multiplication provides an efficient method for reducing the power dissipation and area of rounded parallel multiplier. High speed multiplication is desired in DSP which is normally achieved by parallel processing and pipelining, but by truncation that can be multi fold. The objective of this study is to present a comparative study of truncated and standard multiplier using Spartan-3AN, Virtex-4 and Virtex-5 FPGA devices.

\section{MATERIALS AND METHODS}

Mathematical basis of truncated multipliers: Considering the multiplication of two n-bit inputs $\mathrm{X}$ and $\mathrm{Y}$, a standard multiplier performs the following operations to obtain the $2 \mathrm{n}$ bit product $\mathrm{P}$ :

$$
P=X Y=\sum_{i=0}^{2 n-1} P_{i} 2^{i}=\left(\sum_{i=0}^{n-1} x_{i} 2^{i}\right)\left(\sum_{i=0}^{n-1} y_{i} 2^{i}\right)
$$

where, $x_{i}, y_{i}$ and $P_{i}$ represent the ith bit of $\mathrm{X}, \mathrm{Y}$ and $\mathrm{P}$, respectively. Figure 1 shows the standard architecture of 6×6-bit parallel multiplier, where HA and FA are the half and full adders respectively. Equation 1 can be expressed by the sum of two segments: The mostsignificant part MP and the least-significant part LP:

$$
\mathrm{P}=\mathrm{MP}+\mathrm{LP}=\sum_{\mathrm{i}=0}^{2 \mathrm{n}-1} \mathrm{P}_{\mathrm{i}} 2^{\mathrm{i}}+\sum_{\mathrm{i}=0}^{\mathrm{n}-1} \mathrm{P}_{\mathrm{i}} 2^{\mathrm{i}}
$$

The standard 6×6-bit parallel multiplier can also be divided into three subsets: The most-significant part MP, input correction IC and the least-significant part LP. Equation 2 can be rewritten as follows:

$\mathrm{P}=\mathrm{MP}+\mathrm{IC}+\mathrm{LP}$

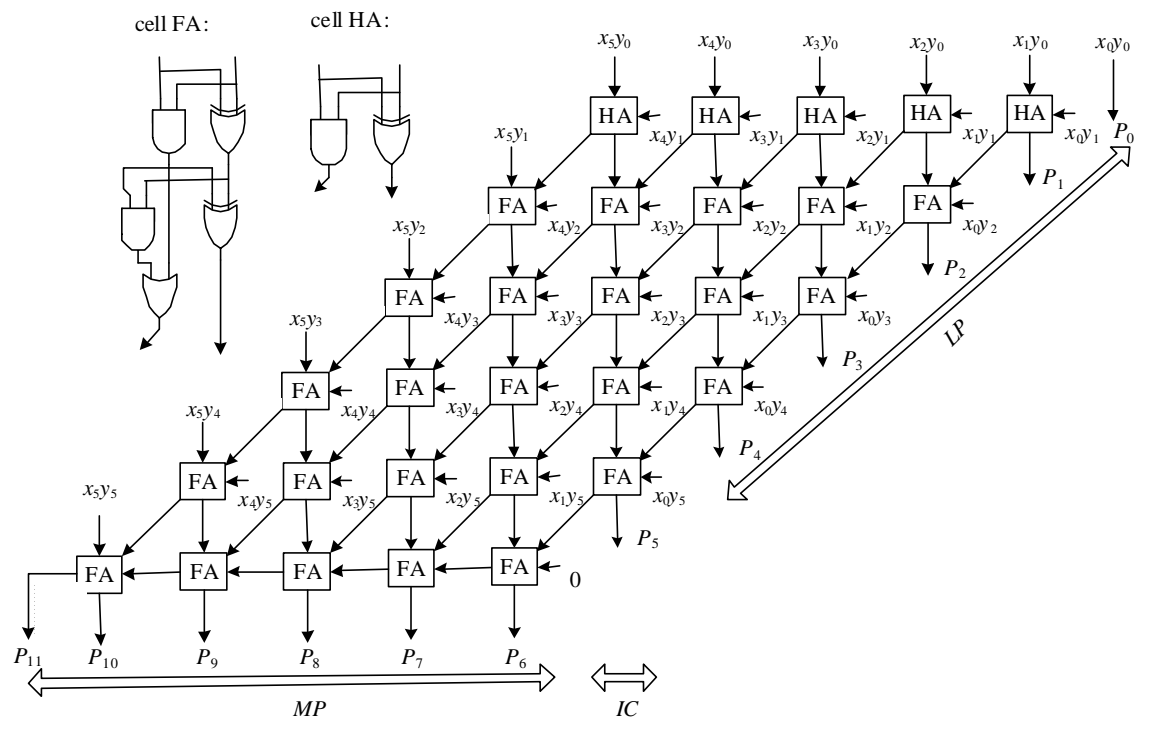

Fig. 1: The architecture of a standard $6 \times 6$-bit parallel multiplier 


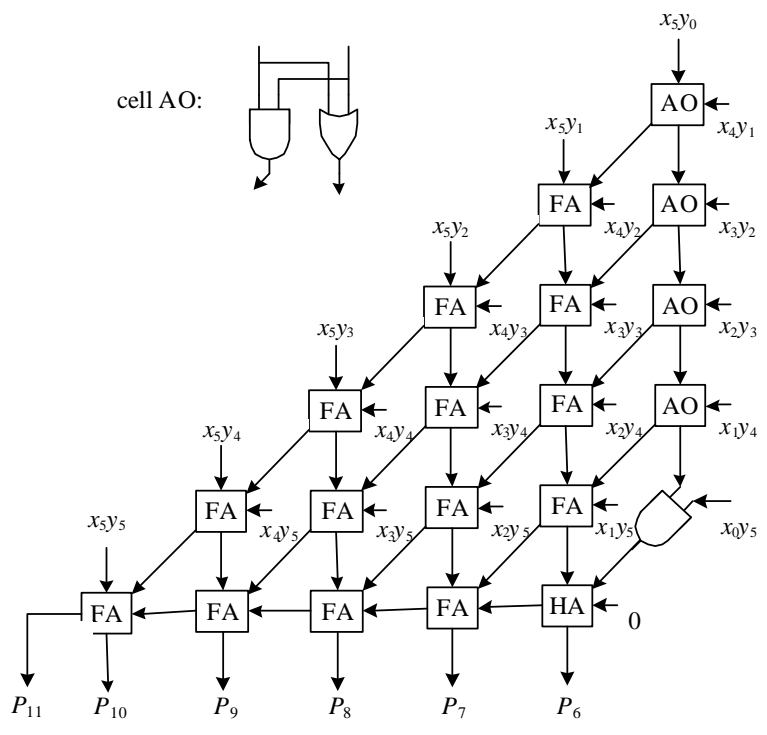

Fig. 2: The architecture of a truncated $6 \times 6$-bit parallel multiplier

The fixed width multiplier can be obtained directly by removing the LP region and introducing the IC region to obtain $\mathrm{MP}^{\prime}$ region, which is truncated multiplier as shown in Fig. 2 and given by Eq. 4:

$\mathrm{P}=\mathrm{MP}^{\prime}+\mathrm{IC}$

Architecture platform: Due to the parallel nature, high frequency and high density of modern FPGAs, they make an ideal platform for the implementation of computationally intensive and massively parallel architecture. A brief introduction about state-of-the-art FPGAs from Xilinx is presented.

Spartan-3 FPGAs: The Spartan-3 FPGA belongs to the fifth generation Xilinx family. It is specifically designed to meet the needs of high volume, low unit cost electronic systems. The family consists of 8 member offering densities ranging from 50,000 to five million system gates (Xilinx, 2008a).

The Spartan-3 FPGA consists of five fundamental programmable functional elements: CLBs, IOBs, Block RAMs, dedicated multipliers $(18 \times 18)$ and Digital Clock Managers (DCMs), Spartan-3 family includes Spartan3L, Spartan-3E, Spartan-3A, Spartan-3A DSP, Spartan3AN and the extended Spartan-3A FPGAs. Particularly, the Spartan-3AN is used as a target technology in this study. Spartan-3AN combines all the feature of Spartan-3A FPGA family plus leading technology insystem flash memory for configuration and nonvolatile data storage.
Virtex-4 FPGAs: Viretx-4 FPGAs are produced on a state-of-the-art $90 \mathrm{~nm}$ copper process, using $300 \mathrm{~nm}$ wafer technology (Xilinx, 2007). It consists of three platform families i.e., LX, SX and FX. Virtex-4 hard-IP core blocks include the IBM Power PC (PPC) 405 32bit Reduced Instruction Set Computer (RISC) processor; tri-mode Ethernet Media Access Controls (MACs) 622 Mbps-6.5 Gbps serial transceivers, dedicated DSP slices and high speed clock management circuitry.

Virtex-4 devices consumes approximately 50\% the power of respective Virtex-II Pro devices due to static and dynamic power reduction enabled by triple-oxide technology and reduced core voltage and capacitance respectively. The Virtex-4 FPGA family comprises of CLBs, Block RAMs, XtremeDSP Slices and DCMs.

Virtex-5 FPGAs: The Virtex-5 devices built on a $65 \mathrm{~nm}$ state-of-the-art copper process technology are a programmable alternative to custom ASIC technology. The Virtex-5 LX platform also contains many hard-IP system-level blocks, including Block RAM/First In First Out (FIFO), second generation $25 \times 18$ DSP slices, SelectIO technology with built-in digitally-controlled impedance, ChipSync source-synchronous interface blocks, enhanced clock management tiles with integrated DCM and Phase Locked Loop (PLL) clock generators and advanced configuration options.

In addition to the regular programmable functional elements, Virtex-5 family provides power-optimized high speed serial transceiver blocks for enhanced serial connectivity, tri-mode Ethernet MACs and highperformance PPC 440 microprocessor embedded blocks. Virtex-5 devices also use triple-oxide technology for reducing the static power consumption. Their $1.0 \mathrm{~V}$ core voltage and $65 \mathrm{~nm}$ implementation process leads also to dynamic power consumption reduction as compared to Virtex-4 devices.

Advanced DSP48E slices are available in Virtex-5 FPGAs that helps in accelerating computation intensive DSP and image processing algorithms. These slices can operate at a maximum frequency of $550 \mathrm{MHz}$, drawing only $1.38 \mathrm{~mW}$ of power at $100 \mathrm{MHz}$ frequency (Xilinx, 2008b).

\section{RESULTS}

FPGA design and implementation results: The design of standard and truncated $4 \times 4,6 \times 6,8 \times 8$ and $12 \times 12$-bit multipliers are done using VHDL and implemented in a Xilinx Spartan-3AN XC3S700AN (package: fgg484, speed grade: -5), Virtex-4 XC4LX40 (package: ff668, speed grade: -12) and Virtex-5 XC5VLX40 (package: ff676, speed grade: -3) FPGAs using the Xilinx ISE 9.2i design tool. 
Am. J. Engg. \& Applied Sci., 3 (1): 201-206, 2010

Table 1: FPGA resource utilization for standard and truncated multiplier for Spartan-3AN XC3S700AN (package: fgg484, speed grade:-5)

\begin{tabular}{llllllll}
\hline $\begin{array}{l}\text { Bit } \\
\text { width }\end{array}$ & Multipliers & $\begin{array}{l}\text { Four-input } \\
\text { LUTs } \\
(11776)\end{array}$ & $\begin{array}{l}\text { Occupied } \\
\text { slices } \\
(5888)\end{array}$ & $\begin{array}{l}\text { Bonded } \\
\text { IOBs } \\
(372)\end{array}$ & $\begin{array}{l}\text { Total } \\
\text { equivalent } \\
\text { gate count }\end{array}$ & $\begin{array}{l}\text { Average } \\
\text { connection } \\
\text { delay (ns) }\end{array}$ & $\begin{array}{l}\text { Maximum } \\
\text { pin delay } \\
(\mathrm{ns})\end{array}$ \\
\hline $4 \times 4$ & Standard & 30 & 16 & 16 & 180 & 1.421 & 3.598 \\
& Truncated & 18 & 11 & 12 & 111 & 1.272 & 2.705 \\
$6 \times 6$ & Standard & 67 & 36 & 24 & 402 & 1.238 & 4.873 \\
& Truncated & 43 & 24 & 18 & 261 & 1.096 & 2.722 \\
& Standard & 121 & 62 & 32 & 726 & 1.085 & 3.968 \\
& Truncated & 76 & 40 & 24 & 456 & 1.072 & 3.641 \\
& Standard & 289 & 148 & 48 & 1734 & 1.079 & 3.766 \\
& Truncated & 164 & 87 & 36 & 984 & 1.307 & 3.971 \\
\hline
\end{tabular}

Table 2: FPGA resource utilization for standard and truncated multiplier for Virtex-4 XC4LX40 (package: ff668, speed grade: -12)

\begin{tabular}{|c|c|c|c|c|c|c|c|}
\hline $\begin{array}{l}\text { Bit } \\
\text { width }\end{array}$ & Multipliers & $\begin{array}{l}\text { Four-input } \\
\text { LUTs } \\
(36864)\end{array}$ & $\begin{array}{l}\text { Occupied } \\
\text { slices } \\
(18432)\end{array}$ & $\begin{array}{l}\text { Bonded } \\
\text { IOBs } \\
(448)\end{array}$ & $\begin{array}{l}\text { Total } \\
\text { equivalent } \\
\text { gate count }\end{array}$ & $\begin{array}{l}\text { Average } \\
\text { connection } \\
\text { delay (ns) }\end{array}$ & $\begin{array}{l}\text { Maximum } \\
\text { pin delay } \\
(\mathrm{ns})\end{array}$ \\
\hline \multirow[t]{2}{*}{$4 \times 4$} & Standard & 30 & 16 & 16 & 180 & 1.014 & 2.225 \\
\hline & Truncated & 18 & 11 & 12 & 111 & 0.954 & 2.137 \\
\hline \multirow[t]{2}{*}{$6 \times 6$} & Standard & 67 & 36 & 24 & 402 & 0.914 & 2.519 \\
\hline & Truncated & 43 & 24 & 18 & 261 & 0.904 & 2.137 \\
\hline \multirow[t]{2}{*}{$8 \times 8$} & Standard & 121 & 63 & 32 & 726 & 0.807 & 2.137 \\
\hline & Truncated & 76 & 40 & 24 & 456 & 0.787 & 2.137 \\
\hline \multirow[t]{2}{*}{$12 \times 12$} & Standard & 289 & 149 & 48 & 1734 & 0.951 & 2.653 \\
\hline & Truncated & 164 & 86 & 36 & 984 & 0.856 & 2.295 \\
\hline
\end{tabular}

Table 3: FPGA resource utilization for standard and truncated multiplier for Virtex-5 XC5VLX50 (package: ff676, speed grade: -3)

\begin{tabular}{|c|c|c|c|c|c|c|c|}
\hline $\begin{array}{l}\text { Bit } \\
\text { width }\end{array}$ & Multipliers & $\begin{array}{l}\text { Four-input } \\
\text { LUTs } \\
(28800)\end{array}$ & $\begin{array}{l}\text { Occupied } \\
\text { slices } \\
(7200)\end{array}$ & $\begin{array}{l}\text { Bonded } \\
\text { IOBs } \\
(440)\end{array}$ & $\begin{array}{l}\text { Total } \\
\text { equivalent } \\
\text { gate count }\end{array}$ & $\begin{array}{l}\text { Average } \\
\text { connection } \\
\text { delay (ns) }\end{array}$ & $\begin{array}{l}\text { Maximum } \\
\text { pin delay } \\
(\mathrm{ns})\end{array}$ \\
\hline \multirow[t]{2}{*}{$4 \times 4$} & Standard & 22 & 15 & 16 & 154 & 0.927 & 1.990 \\
\hline & Truncated & 11 & 6 & 12 & 77 & 0.773 & 1.140 \\
\hline \multirow[t]{2}{*}{$6 \times 6$} & Standard & 46 & 28 & 24 & 322 & 0.955 & 1.757 \\
\hline & Truncated & 27 & 14 & 18 & 182 & 0.854 & 1.730 \\
\hline \multirow[t]{2}{*}{$8 \times 8$} & Standard & 83 & 30 & 32 & 581 & 0.826 & 1.697 \\
\hline & Truncated & 50 & 19 & 24 & 350 & 0.765 & 1.500 \\
\hline \multirow[t]{2}{*}{$12 \times 12$} & Standard & 190 & 88 & 48 & 1330 & 1.107 & 2.845 \\
\hline & Truncated & 117 & 65 & 36 & 819 & 1.027 & 2.611 \\
\hline
\end{tabular}

Figure 3 shows the comparison of number of occupied slices for Spartan-3AN, Virtex-4 and Virtex-5 FPGA devices for standard and truncated multipliers, which clearly indicates that the virtex-5 FPGA device utilizes fewer resources than Spartan-3AN and Virtex-4 devices.

Table 1-3 summarize the FPGA devices resources utilization for standard and truncated $4 \times 4,6 \times 6,8 \times 8$ and $12 \times 12$ bit multipliers.

The four input Look-Up-Tables (LUTs) for standard and truncated multipliers for Spartan-3AN, Virtex-4 and Virtex-5 FPGA devices are shown in Fig. 4. The same phenomenon is observed here as compared to number of occupied slices for all the devices. Figure 5 illustrates the total equivalent gate count obtained for standard and truncated multipliers for Spartan-3AN, Virtex-4 and Virtex-5 FPGA devices.

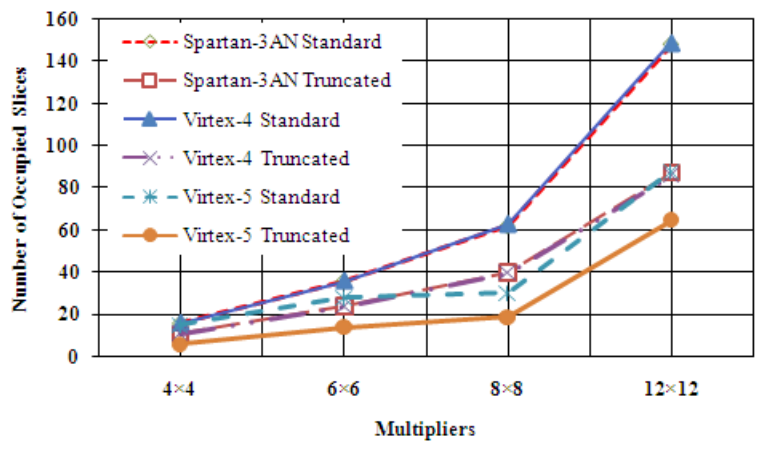

Fig. 3: The number of occupied slices for Spartan3AN, Virtex-4 and Virtex-5 for standard and truncated multipliers

Figure 6 and 7 show the average connection delay and maximum pin delay for Spartan-3AN, Virtex-4 and Virtex-5 FPGA devices for standard and truncated multipliers respectively. 


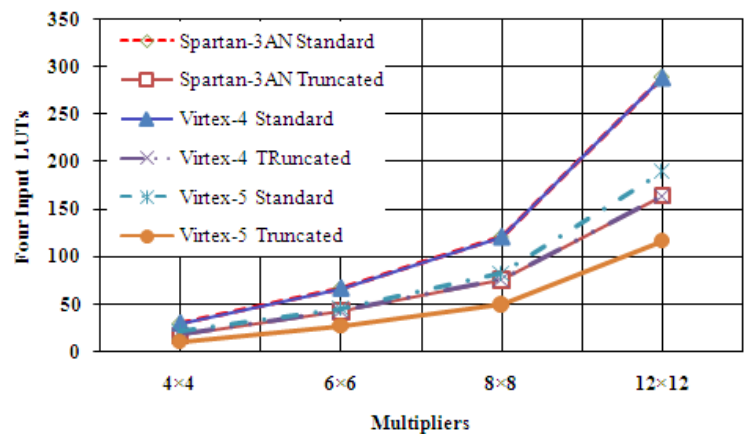

Fig. 4: The four input LUTs for Spartan-3AN, Virtex-4 and Virtex-5 for standard and truncated multipliers

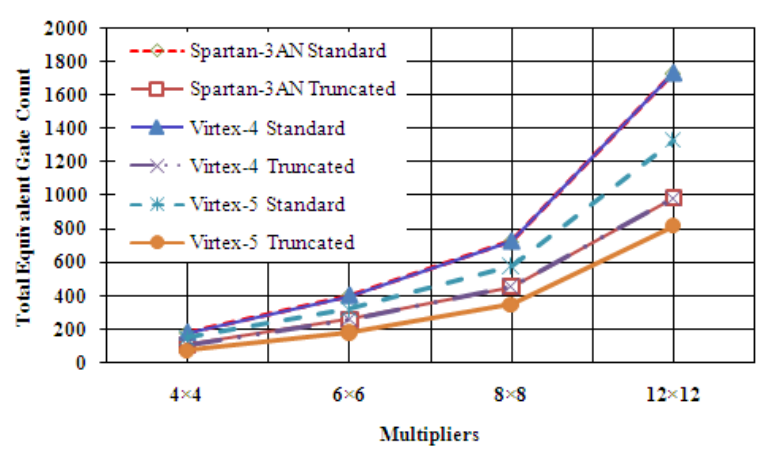

Fig. 5: The total equivalent gate count for Spartan3AN, Virtex-4 and Virtex-5 for standard and truncated multipliers

\section{DISCUSSION}

The Virtex-5 FPGA device shows better performance than Virtex-4 and Spartan-3AN FPGA devices with a percentage ratio of occupied slices for standard to truncated multipliers is increased from $40 \%$ to $73.86 \%$ as compared to Spartan-3AN and Virtex-4 FPGA devices is decreased from $68.75-58.78 \%$. Another important feature is also visible in Fig. 3 is that the Virtex-5 FPGA device almost uses same resources for standard multiplier as the truncated multiplier for the Spartan-3AN and Virtex-4 FPGA devices.

The abnormality in average connection delay and maximum pin delay for Spartan-3AN device (12×12-bit multiplier for standard and truncated multiplier) has been significantly reduced in Virtex 4 and Virtex-5 FPGA devices as shown in Fig. 6 and 7, Table 1-3 respectively.

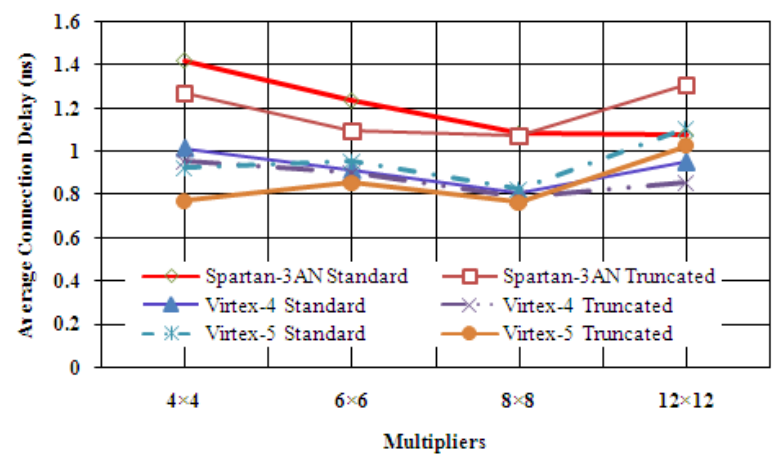

Fig. 6: The average connection delay for Spartan-3AN, Virtex-4 and Virtex-5 for standard and truncated multipliers

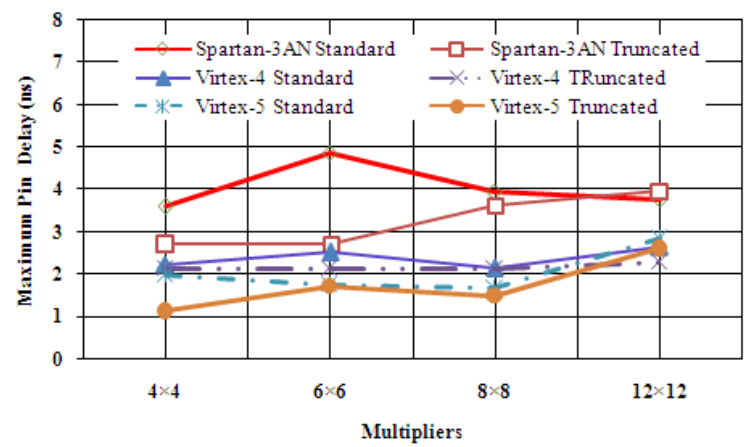

Fig. 7: The maximum pin delay for Spartan-3AN, Virtex-4 and Virtex-5 for standard and truncated multipliers

\section{CONCLUSION}

In this study we have presented hardware design and implementation of FPGA based parallel architecture for standard and truncated multipliers utilizing VHDL. The design was implemented on Xilinx Spartan-3AN XC3S700AN, Virtex-4 XC4LX40 and Virtex-5 XC5VLX40 FPGA devices using the ISE $9.2 \mathrm{i}$ design tool. The objective is to present a comparative study of the standard and truncated multipliers. The truncated multiplier shows much more reduction in device utilization as compared to standard multiplier. The standard and truncated multipliers shows that the average connection delay and maximum pin delay have been significantly reduced in Virtx-4 and Virtex-5 FPGA devices. The Viretx-5 FPGA devices achieves better result than Spartan-3AN and Virtex-4 FPGA device and is a viable FPGA device for image processing, multimedia and digital signal processing. 


\section{ACKNOWLEDGEMENT}

The researcher acknowledges the assistance and the financial support provided by the Research Center in the College of Engineering, King Saud University vide their Research Grant No. 14/431.

\section{REFERENCES}

Baugh, C.R. and B.A. Wooley, 1973. A two's complement parallel array multiplication algorithm. IEEE Trans. Comput., C-22:12: 1045-1047.

Garofalo, V., N. Petra, D. DeCaro, A.G.M. Strollo and E. Napoli, 2008. Low error truncated multipliers for DSP applications. Proceedings of the 15th IEEE International Conference on Electronics, Circuits and Systems, Aug. 31-Sept. 3, IEEE Xplore Press, USA., pp: 29-32. DOI: 10.1109/ICECS.2008.4674783

Jou, J.M., S.R. Kuang and R.D. Chen, 1999. Design of low-error fixed-width multipliers for DSP applications. IEEE Trans. Circ. Syst.-II: Analog Digital Signal Process., 46: 836-842. DOI: 10.1109/82.769795

Kidambi, S.S., F. El-Guibaly and A. Antonious, 1996. Area-efficient multipliers for digital signal processing applications. IEEE Trans. Circ. Syst.-II: Analog Digital Signal Process., 43: 90-95. DOI: 10.1109/82.486455

Kuang, S.R. and J.P. Wang, 2006, Low-error configurable truncated multipliers for multiplyaccumulate applications. Elect. Lett., 42: 904-905. DOI: $10.1049 / \mathrm{el}: 20061812$

Lim, Y.C., 1992. Single-precision multiplier with reduced circuit complexity for signal processing applications. IEEE Trans. Comput., 41: 1333-1336. DOI: $10.1109 / 12.166611$

Maxfield, C., 2004. The Design Warrior's Guide to FPGAs: Devices, Tools and Flows. Newnes Publishers, MA., USA., ISBN: 0750676043, pp: 121-131.

Rais, M.H., 2009a. FPGA design and implementation of fixed width standard and truncated 6×6-bit multipliers: A comparative study. Proceedings of the 4th IEEE International Design and Test Workshop, Nov. 15-17, IEEE Xplore Press, Riyadh, Saudi Arabia, pp: 1-4. DOI: 10.1109/IDT.2009.5404081

Rais, M.H., 2009b. Efficient hardware realization of truncated multipliers using FPGA. Int. J. Applied Sci. Eng. Technol., 5: 124-128. http://www.waset.org/journals/ijaset/v5/v5-218.pdf
Sheu, M.H. and S.H. Lin, 2002. Fast compensative design approach of the approximate squaring function. IEEE J. Solid State Circ., 37: 95-97. DOI: $10.1109 / 4.974551$

Stallings, W., 2006. Cryptography and Network Security: Principles and Practices. 4th Edn., Prentice-Hall, Upper Saddle River, New Jersey, ISBN: 013 1873164, pp: 97-119

Stine, J.E. and O.M. Duverne, 2003. Variations on truncated multiplication. Proceedings of the Euromicro Symposium on Digital System Design, Sept. 1-6, IEEE Computer Society, Washington DC., $\quad$ USA., $\quad$ pp: 112-119. http://portal.acm.org/citation.cfm?id=943082

Strollo, A.G.M., N. Petra and D. DeCaro, 2005. Dualtree error compensation for high performance fixed-width multipliers. IEEE Trans. Circ. Syst.-II: Analog Digital Signal Process., 52: 501-507. DOI: 10.1109/TCSII.2005.848979

Swartzlander Jr., E.E., 1999. Truncated multiplication with approximate rounding. Proceedings of the 33rd Asilomar Conference on Signals, Systems and Computers, Oct. 24-27, IEEE Xplore Press, Pacific Grove, CA., USA., pp: 1480-1483. DOI: 10.1109/ACSSC.1999.831996

Todman, T.J., G.A. Constantinides, S.J.E. Wilton, O. Mencer, W. Luk and P.Y.K. Cheung, 2005. Reconfigurable computing: architectures and design methods. IEE Proc. Comput. Digit. Tech., 152: 193-207. DOI: 10.1049/ip-cdt:20045086

Van, L.D., S.S. Wang and W.S. Feng, 2000. Design of the lower error fixed-width multiplier and its application. IEEE Trans. Circ. Syst.-II: Analog Digit. Sign. Process., 47: 1112-1118. DOI: 10.1109/82.877155

Walters, E., M.G. Arnold and M.J. Schulte, 2003. Using truncated multipliers in DCT and IDCT hardware accelerators. Proceedings of the XIII SPIE Advanced Signal Processing Algorithms, Architectures and Implementations, Aug. 6-8, San International Society for Optical Engineering, San Diego, California, pp: 573-584.

Xilinx, 2007. Virtex-4 FPGA family overview. http://www.xilinx.com/support/documentation/data _sheets/ds112.pdf

Xilinx, 2008a. Spartan-3 FPGA family datasheet. http://www.xilinx.com/support/documentation/spar $\tan -3 \mathrm{e} . \mathrm{htm}$

Xilinx, 2008b. Virtex-5 FPGA family overview. http://www.xilinx.com/support/documentation/virt ex-5.htm 